FACTA UNIVERSITATIS (NIŠ)

Ser. Math. Inform. Vol. 35, No 4 (2020), 1199-1204

https://doi.org/10.22190/FUMI2004199B

\title{
A NEW STUDY ON ABSOLUTE CESÀRO SUMMABILITY FACTORS
}

\author{
Hüseyin Bor
}

(C) 2020 by University of Niš, Serbia | Creative Commons Licence: CC BY-NC-ND

Abstract. In this paper, we have generalized a known theorem dealing with $\varphi-|C, \alpha,|_{k}$ summability factors of infinite series to the $\varphi-|C, \alpha, \beta|_{k}$ summability method under weaker conditions. Also, some new and known results have been obtained.

Keywords: summability factors; infinite series; Cesàro mean; Hölder's inequality; Minkowsk's inequality; almost increasing sequences.

\section{Introduction}

A positive sequence $\left(b_{n}\right)$ is said to be almost increasing sequence if there exists a positive increasing sequence $\left(c_{n}\right)$ and two positive constants $M$ and $N$ such that $M c_{n} \leq b_{n} \leq N c_{n}$ (see [2]). Let $\sum a_{n}$ be a given infinite series. We denote by $t_{n}^{\alpha, \beta}$ the $n$th Cesàro mean of order $(\alpha, \beta)$, with $\alpha+\beta>-1$, of the sequence $\left(n a_{n}\right)$, that is (see [8])

$$
t_{n}^{\alpha, \beta}=\frac{1}{A_{n}^{\alpha+\beta}} \sum_{v=1}^{n} A_{n-v}^{\alpha-1} A_{v}^{\beta} v a_{v}
$$

where

$$
A_{n}^{\alpha+\beta}=O\left(n^{\alpha+\beta}\right), \quad A_{0}^{\alpha+\beta}=1 \quad \text { and } \quad A_{-n}^{\alpha+\beta}=0 \quad \text { for } n>0 .
$$

Let $\left(\omega_{n}^{\alpha, \beta}\right)$ be a sequence defined by (see [5])

$$
\omega_{n}^{\alpha, \beta}=\left\{\begin{array}{cc}
\left|t_{n}^{\alpha, \beta}\right|, & \alpha=1, \beta>-1 \\
\max _{1 \leq v \leq n}\left|t_{v}^{\alpha, \beta}\right|, & 0<\alpha<1, \beta>-1 .
\end{array}\right.
$$

Received December 28, 2019; accepted March 27, 2020

2020 Mathematics Subject Classification. Primary 40D15, 26D15 ; Secondary 40F05, 40G05 
Let $\left(\varphi_{n}\right)$ be a sequence of complex numbers. The series $\sum a_{n}$ is said to be summable $\varphi-|C, \alpha, \beta|_{k}, k \geq 1$, if (see [6])

$$
\sum_{n=1}^{\infty} n^{-k}\left|\varphi_{n} t_{n}^{\alpha, \beta}\right|^{k}<\infty
$$

In the special case when $\varphi_{n}=n^{1-\frac{1}{k}}, \varphi-|C, \alpha, \beta|_{k}$ summability is the same as $|C, \alpha, \beta|_{k}$ summability (see [9]). Also, if we take $\varphi_{n}=n^{\delta+1-\frac{1}{k}}$, then $\varphi-|C, \alpha, \beta|_{k}$ summability reduces to $|C, \alpha, \beta ; \delta|_{k}$ summability (see [7]). If we take $\beta=0$, then we have $\varphi-|C, \alpha|_{k}$ summability (see [1]). If we take $\varphi_{n}=n^{1-\frac{1}{k}}$ and $\beta=0$, then we get $|C, \alpha|_{k}$ summability (see [10]). Finally, if we take $\varphi_{n}=n^{\delta+1-\frac{1}{k}}$ and $\beta=0$, then we obtain $|C, \alpha ; \delta|_{k}$ summability (see [11]).

\section{Known Result}

The following theorem is known dealing with the $\varphi-|C, \alpha|_{k}$ summability factors of infinite series.

Theorem 2.1 ([3]). Let $0<\alpha \leq 1$. Let $\left(X_{n}\right)$ be a positive non-decreasing sequence and let there be sequences $\left(\beta_{n}\right)$ and $\left(\lambda_{n}\right)$ such that

$$
\begin{gathered}
\left|\Delta \lambda_{n}\right| \leq \beta_{n} \\
\beta_{n} \rightarrow 0 \quad \text { as } n \rightarrow \infty \\
\sum_{n=1}^{\infty} n\left|\Delta \beta_{n}\right| X_{n}<\infty \\
\left|\lambda_{n}\right| X_{n}=O(1) \text { as } n \rightarrow \infty .
\end{gathered}
$$

If there exists an $\epsilon>0$ such that the sequence $\left(n^{\epsilon-k}\left|\varphi_{n}\right|^{k}\right)$ is non increasing and if the sequence $\left(\omega_{n}^{\alpha}\right)$ defined by (see [13])

$$
\omega_{n}^{\alpha}=\left\{\begin{array}{cc}
\left|t_{n}^{\alpha}\right| & (\alpha=1) \\
\max _{1 \leq v \leq n}\left|t_{v}^{\alpha}\right| & (0<\alpha<1)
\end{array}\right.
$$

satisfies the condition

$$
\sum_{n=1}^{m} n^{-k}\left(\left|\varphi_{n}\right| \omega_{n}^{\alpha}\right)^{k}=O\left(X_{m}\right) \quad \text { as } \quad m \rightarrow \infty
$$

then the series $\sum a_{n} \lambda_{n}$ is summable $\varphi-|C, \alpha|_{k}, k \geq 1$ and $(\alpha+\epsilon)>1$. 


\section{Main Result}

The aim of this paper is to generalize Theorem 2.1 for $\varphi-|C, \alpha, \beta|_{k}$ summability method under weaker conditions by using an almost increasing sequence instead of a positive non-decreasing sequence. Now we shall prove the following theorem.

Theorem 3.1. Let $0<\alpha \leq 1$ and let $\left(X_{n}\right)$ be an almost increasing sequence. Let there be sequences $\left(\beta_{n}\right)$ and $\left(\lambda_{n}\right)$ such that conditions (2.1)-(2.4) of Theorem 2.1 are satisfied. If there exists an $\epsilon>0$ such that the sequence $\left(n^{\epsilon-k}\left|\varphi_{n}\right|^{k}\right)$ is non increasing and if the sequence $\left(\omega_{n}^{\alpha, \beta}\right)$ defined by (1.3), satisfies the condition

$$
\sum_{n=1}^{m} n^{-k}\left(\left|\varphi_{n}\right| \omega_{n}^{\alpha, \beta}\right)^{k}=O\left(X_{m}\right) \quad \text { as } \quad m \rightarrow \infty,
$$

then the series $\sum a_{n} \lambda_{n}$ is summable $\varphi-|C, \alpha, \beta|_{k}, k \geq 1,0<\alpha \leq 1, \beta>-1$, and $(\alpha+\beta) k+\epsilon>1$.

Remark. It should be noted that, obviously every increasing sequence is almost increasing. However, the converse need not be true (see [12]).

We need the following lemmas for the proof of our theorem.

Lemma 3.1 ([5]). If $0<\alpha \leq 1, \beta>-1$, and $1 \leq v \leq n$, then

$$
\left|\sum_{p=0}^{v} A_{n-p}^{\alpha-1} A_{p}^{\beta} a_{p}\right| \leq \max _{1 \leq m \leq v}\left|\sum_{p=0}^{m} A_{m-p}^{\alpha-1} A_{p}^{\beta} a_{p}\right| .
$$

Lemma $3.2([4])$. Under the conditions on $\left(X_{n}\right),\left(\beta_{n}\right)$ and $\left(\lambda_{n}\right)$ as taken in the statement of the theorem, the following conditions hold, when (2.3) is satisfied

$$
\begin{gathered}
n \beta_{n} X_{n}=O(1) \text { as } n \rightarrow \infty \\
\sum_{n=1}^{\infty} \beta_{n} X_{n}<\infty .
\end{gathered}
$$

4. Proof of Theorem 3.1. Let $\left(T_{n}^{\alpha, \beta}\right)$ be the $n$th $(C, \alpha, \beta)$ mean of the sequence $\left(n a_{n} \lambda_{n}\right)$. Then, by (1.1), we have

$$
T_{n}^{\alpha, \beta}=\frac{1}{A_{n}^{\alpha+\beta}} \sum_{v=1}^{n} A_{n-v}^{\alpha-1} A_{v}^{\beta} v a_{v} \lambda_{v} .
$$

Applying Abel's transformation first and then using Lemma 3.1, we have that

$$
\begin{aligned}
T_{n}^{\alpha, \beta} & =\frac{1}{A_{n}^{\alpha+\beta}} \sum_{v=1}^{n-1} \Delta \lambda_{v} \sum_{p=1}^{v} A_{n-p}^{\alpha-1} A_{p}^{\beta} p a_{p}+\frac{\lambda_{n}}{A_{n}^{\alpha+\beta}} \sum_{v=1}^{n} A_{n-v}^{\alpha-1} A_{v}^{\beta} v a_{v} \\
\left|T_{n}^{\alpha, \beta}\right| & \leq \frac{1}{A_{n}^{\alpha+\beta}} \sum_{v=1}^{n-1}\left|\Delta \lambda_{v}\right|\left|\sum_{p=1}^{v} A_{n-p}^{\alpha-1} A_{p}^{\beta} p a_{p}\right|+\frac{\left|\lambda_{n}\right|}{A_{n}^{\alpha+\beta}}\left|\sum_{v=1}^{n} A_{n-v}^{\alpha-1} A_{v}^{\beta} v a_{v}\right| \\
\leq & \frac{1}{A_{n}^{\alpha+\beta}} \sum_{v=1}^{n-1} A_{v}^{(\alpha+\beta)} \omega_{v}^{\alpha, \beta}\left|\Delta \lambda_{v}\right|+\left|\lambda_{n}\right| \omega_{n}^{\alpha, \beta}=T_{n, 1}^{\alpha, \beta}+T_{n, 2}^{\alpha, \beta} .
\end{aligned}
$$


To complete the proof of the theorem 3.1, by Minkowski's inequality, it is sufficient to show that

$$
\sum_{n=1}^{\infty} n^{-k}\left|\varphi_{n} T_{n, r}^{\alpha, \beta}\right|^{k}<\infty, \quad \text { for } \quad r=1,2 .
$$

Now, when $k>1$, applying Hölder's inequality with indices $k$ and $k^{\prime}$, where $\frac{1}{k}+\frac{1}{k^{\prime}}=$ 1 , we get that

$$
\begin{aligned}
& \sum_{n=2}^{m+2} n^{-k}\left|\varphi_{n} T_{n, 1}^{\alpha, \beta}\right|^{k} \leq \sum_{n=2}^{m+1} n^{-k}\left(A_{n}^{\alpha+\beta}\right)^{-k}\left|\varphi_{n}\right|^{k}\left\{\sum_{v=1}^{n-1} A_{v}^{\alpha+\beta} \omega_{v}^{\alpha, \beta}\left|\Delta \lambda_{v}\right|\right\}^{k} \\
& \leq \sum_{n=2}^{m+1} n^{-k} n^{-(\alpha+\beta) k}\left|\varphi_{n}\right|^{k} \sum_{v=1}^{n-1}\left(A_{v}^{\alpha+\beta}\right)^{k}\left(\omega_{v}^{\alpha, \beta}\right)^{k} \beta_{v} \cdot\left\{\sum_{v=1}^{n-1} \beta_{v}\right\}^{k-1} \\
& =O(1) \sum_{v=1}^{m} v^{(\alpha+\beta) k}\left(\omega_{v}^{\alpha, \beta}\right)^{k} \beta_{v} \sum_{n=v+1}^{m+1} \frac{n^{\epsilon-k}\left|\varphi_{n}\right|^{k}}{n^{(\alpha+\beta) k+\epsilon}} \\
& =O(1) \sum_{v=1}^{m} v^{(\alpha+\beta) k}\left(\omega_{v}^{\alpha, \beta}\right)^{k} \beta_{v} v^{\epsilon-k}\left|\varphi_{v}\right|^{k} \sum_{n=v+1}^{m+1} \frac{1}{n^{(\alpha+\beta) k+\epsilon}} \\
& =O(1) \sum_{v=1}^{m} v^{(\alpha+\beta) k}\left(\omega_{v}^{\alpha, \beta}\right)^{k} v^{\epsilon-k}\left|\varphi_{v}\right|^{k} \beta_{v} \int_{v}^{\infty} \frac{d x}{x^{(\alpha+\beta) k+\epsilon}} \\
& =O(1) \sum_{v=1}^{m} v \beta_{v} v^{-k}\left(\omega_{v}^{\alpha, \beta}\left|\varphi_{v}\right|\right)^{k} \\
& =O(1) \sum_{v=1}^{m-1} \Delta\left(v \beta_{v}\right) \sum_{r=1}^{v} r^{-k}\left(\omega_{r}^{\alpha, \beta}\left|\varphi_{r}\right|\right)^{k} \\
& +O(1) m \beta_{m} \sum_{v=1}^{m} v^{-k}\left(\omega_{v}^{\alpha, \beta}\left|\varphi_{v}\right|\right)^{k} \\
& =O(1) \sum_{v=1}^{m-1}\left|\Delta\left(v \beta_{v}\right)\right| X_{v}+O(1) m \beta_{m} X_{m} \\
& =O(1) \sum_{v=1}^{m-1} v\left|\Delta \beta_{v}\right| X_{v}+O(1) \sum_{v=1}^{m-1} \beta_{v} X_{v}+O(1) m \beta_{m} X_{m} \\
& =O(1) \text { as } m \rightarrow \infty,
\end{aligned}
$$

by the hypotheses of Theorem 3.1 and Lemma 3.2. Since, $\left|\lambda_{n}\right|=O(1)$ by (2.4), finally we have that

$$
\begin{aligned}
& \sum_{n=1}^{m} n^{-k}\left|\varphi_{n} T_{n, 2}^{\alpha, \beta}\right|^{k}=O(1) \sum_{n=1}^{m}\left|\lambda_{n}\right|\left|\lambda_{n}\right|^{k-1} n^{-k}\left(\omega_{n}^{\alpha, \beta}\left|\varphi_{n}\right|\right)^{k} \\
= & O(1)) \sum_{n=1}^{m-1} \Delta\left|\lambda_{n}\right| \sum_{v=1}^{n} v^{-k}\left(\omega_{v}^{\alpha, \beta}\left|\varphi_{v}\right|\right)^{k}
\end{aligned}
$$




$$
\begin{aligned}
& +O(1)\left|\lambda_{m}\right| \sum_{n=1}^{m} n^{-k}\left(\omega_{n}^{\alpha, \beta}\left|\varphi_{n}\right|\right)^{k}=O(1) \sum_{n=1}^{m-1}\left|\Delta \lambda_{n}\right| X_{n}+O(1)\left|\lambda_{m}\right| X_{m} \\
= & O(1) \sum_{n=1}^{m-1} \beta_{n} X_{n}+O(1)\left|\lambda_{m}\right| X_{m}=O(1) \quad \text { as } \quad m \rightarrow \infty,
\end{aligned}
$$

by the hypotheses of Theorem 3.1 and Lemma 3.2. This completes the proof of Theorem 3.1. If we take $\epsilon=1$ and $\varphi_{n}=n^{1-\frac{1}{k}}$, then we obtain a new result concerning the $|C, \alpha, \beta|_{k}$ summability factors of infinite series. If we take $\epsilon=1$, $\beta=0$ and $\varphi_{n}=n^{\delta+1-\frac{1}{k}}$, then we have a new result dealing with the $|C, \alpha ; \delta|_{k}$ summability factors of infinite series. Also, if we take $\left(X_{n}\right)$ as a positive nondecreasing sequence and $\beta=0$, then we obtain Theorem 2.1.

\section{REF EREN CES}

1. M. Balc1: Absolute $\varphi$-summability factors. Comm. Fac. Sci. Univ. Ankara, Ser. $A_{1}$, 29, 1980, 63-68.

2. N. K. Bari and S. B. Stečkin: Best approximation and differential properties of two conjugate functions. Trudy. Moskov. Mat. Obšč., 5, 1956, 483-522 (in Russian)

3. H. Bor: Factors for generalized absolute Cesàro summability methods. Publ. Math. Debrecen, 43, 1993, 297-302.

4. H. Bor: An application of almost increasing sequences. Int. J. Math. Math. Sci., 23, 2000, 859-863.

5. H. Bor: On a new application of quasi power increasing sequences. Proc. Est. Acad. Sci., 57, 2008, 205-209.

6. H. Bor: A newer application of almost increasing sequences. Pac. J. Appl. Math., 2, 2010, 211-216.

7. H. Bor: An application of almost increasing sequences. Appl. Math. Lett., 24, 2011, 298-301.

8. D. Borwein: Theorems on some methods of summability. Quart. J. Math. Oxford Ser. (2), 9, 1958, 310-316.

9. G. A. Das: Tauberian theorem for absolute summability. Proc. Camb. Phil. Soc., 67, 1970, 32-326.

10. T. M. Flett: On an extension of absolute summability and some theorems of Littlewood and Paley. Proc. London Math. Soc., 7, 1957, 113-141.

11. T. M. Flett: Some more theorems concerning the absolute summability of Fourier series. Proc. London Math. Soc., 8 1958, 357-387.

12. S. M. Mazhar: Absolute summability factors of infinite series. Kyungpook Math. J., 39, 1999, 67-73.

13. T. Pati: The summability factors of infinite series. Duke Math. J., 1954, 21, 271-284. 
Hüseyin Bor

P. O. Box 121 , TR-06502 Bahçelievler Ankara,Turkey

hbor33@gmail.com 